\title{
UNCONDITIONAL CONVERGENCE AND BASES
}

\author{
by I. TWEDDLE \\ (Received 23rd January 1973)
}

\section{Introduction}

The recent papers (6), (7) of J. T. Marti have revived interest in the concept of extended bases, introduced in (1) by M. G. Arsove and R. E. Edwards. In the present note, two results are established which involve this idea. The first of these, which is given in a more general setting, restricts the behaviour of the coefficients for an extended basis in a certain type of locally convex space. The second result extends the well-known weak basis theorem (1, Theorem 11).

In general, the topological vector space notation of $(\mathbf{1 0})$ is followed. Some results on unconditional convergence are required from (8), particular cases of which are given in the more accessible reference (9) with an indication of how the general results may be obtained. In each case, both references are cited.

\section{Results}

Let $E$ be a separated locally convex space with dual space $E^{\prime} . \quad E$ is said to satisfy condition $\left({ }^{*}\right)$ (respectively condition $\left({ }^{* *}\right)$ ) if each $\beta\left(E^{\prime}, E\right)$ - (respectively $\sigma\left(E^{\prime}, E\right)$-) bounded sequence forms an equicontinuous set. Condition (*), which is a familiar condition in basis theory, is satisfied by quasibarrelled spaces, $(D F)$-spaces and generally, by the countably quasibarrelled spaces of (3). Condition $\left({ }^{* *}\right)$ is the defining condition for the $\omega$-barrelled spaces of (5) and the $\sigma$-barrelled spaces of (2).

Theorem 1. Let $E$ be a separated locally convex space with dual space $E^{\prime}$. Suppose that $E$ satisfies condition $\left({ }^{*}\right)$ and that there is a family $\left(x_{\lambda}\right)_{\lambda \in \Lambda}$ of elements of $E$ such that

(a) for each $x \in E$ there exist scalars $\alpha_{\lambda}(\lambda \in \Lambda)$ such that $\Sigma \alpha_{\lambda} x_{\lambda}$ is unconditionally convergent to $x$,

(b) there is an element of $E$ for which these scalars may all be chosen to be non-zero.

Then $E$ is quasibarrelled under $\tau\left(E, E^{\prime}\right)$.

Proof. Let $F$ be the completion of $E$ and let $F^{\prime}$ be the $\tau\left(E^{\prime}, F\right)$-completion of $E^{\prime}$. Denote the topology of $E$ by $\xi$ and let $\eta$ be its extension to $F$. Let $B$ be a $\sigma\left(E^{\prime}, E\right)$-closed absolutely convex $\beta\left(E^{\prime}, E\right)$-bounded set. It is required to show that $B$ is $\sigma\left(E^{\prime}, E\right)$-compact. If $\left(x_{n}^{\prime}\right)$ is any sequence in $B,\left\{x_{n}^{\prime}: n=1,2, \ldots\right\}$ is equicontinuous under $\xi$ and therefore also under $\eta$. ( $\left.x_{n}^{\prime}\right)$ must therefore have a $\sigma\left(E^{\prime}, F\right)$-cluster point, from which it follows that $B$, being also $\sigma\left(E^{\prime}, F\right)$-closed, 
is $\sigma\left(E^{\prime}, F\right)$-countably compact. Eberlein's theorem now shows that $B$ is $\sigma\left(F^{\prime}, F\right)$-relatively compact. Let $y^{\prime}$ be an element of the $\sigma\left(F^{\prime}, F\right)$-closure of $B$.

Choose an element $z=\sum_{\lambda \in \Lambda} \beta_{\lambda} x_{\lambda}$ of $E$ such that for all $\lambda \in \Lambda, \beta_{\lambda} \neq 0$, and let $A$ be the $\eta$-closed absolutely convex envelope of $\left\{\sum_{\lambda \in \phi} \beta_{\lambda} x_{\lambda}: \phi \in \Phi\right\}$, where $\Phi$ denotes the set of finite subsets of $\Lambda$. Since $A$ is $\eta$-compact (8, Chapter II, Theorem 1; 9, Theorem 1), it is compact under $\sigma\left(F, E^{\prime}\right)$ and therefore also under $\sigma\left(F, F^{\prime}\right)(\mathbf{1 0}$, Chapter VI, Corollaries 3 and 4 of Theorem 2).

Let $M$ be the vector subspace of $F$ generated by $A$ and let $M^{\prime}=F^{\prime} / M^{0}$. Now $\left(M^{\prime}, M\right)$ is a dual pair and $\sigma\left(F, F^{\prime}\right)$ induces $\sigma\left(M, M^{\prime}\right)$ on $M . M^{\prime}$ is therefore a normed space under $\tau\left(M^{\prime}, M\right)$, since $A$ is a $\sigma\left(M, M^{\prime}\right)$-compact absolutely convex and absorbent subset of $M$. The quotient map $q: F^{\prime} \rightarrow M^{\prime}$ is clearly continuous under $\sigma\left(F^{\prime}, F\right)$ and $\sigma\left(M^{\prime}, M\right)$. Thus $q(B)$ is $\sigma\left(M^{\prime}, M\right)$-compact (4, Section 24, 3(9); the result is given for a Fréchet space, but clearly holds for any metrisable locally convex space, since a weakly countably compact set has the same property in the completion of the space). In particular $q(B)$ is $\sigma\left(M^{\prime}, M\right)$-closed, which implies that $q\left(y^{\prime}\right) \in q(B)$; equivalently, there is an element $x^{\prime}$ of $B$ such that $\left\langle x, x^{\prime}\right\rangle=\left\langle x, y^{\prime}\right\rangle$ for all $x \in M$. But $x_{\lambda} \in M$ for all $\lambda \in \Lambda$, since $\beta_{\lambda} x_{\lambda} \in A$ and $\beta_{\lambda} \neq 0$. Hence

$$
\left\langle x_{\lambda}, x^{\prime}\right\rangle=\left\langle x_{\lambda}, y^{\prime}\right\rangle \text { for all } \lambda \in \Lambda \text {. }
$$

Now let $x=\sum_{\lambda \in \Lambda} \alpha_{\lambda} x_{\lambda} \in E$. Since $y^{\prime}$ is $\sigma\left(F, E^{\prime}\right)$-continuous on the $\eta$-closed absolutely convex envelope of $\left\{\sum_{\lambda \in \phi} \alpha_{\lambda} x_{\lambda}: \phi \in \Phi\right\}$ (10, Chapter VI, Theorem 3),

$$
\left\langle x, y^{\prime}\right\rangle=\sum_{\lambda \in \Lambda} \alpha_{\lambda}\left\langle x_{\lambda}, y^{\prime}\right\rangle=\sum_{\lambda \in \Lambda} \alpha_{\lambda}\left\langle x_{\lambda}, x^{\prime}\right\rangle=\left\langle x, x^{\prime}\right\rangle
$$

by $(I)$; i.e. $x^{\prime}$ and $y^{\prime}$ coincide on $E$.

Finally, let $\bar{B}$ be the $\sigma\left(F^{\prime}, F\right)$-closure of $B$ and let $p$ be the quotient map of $F^{\prime}$ onto $F^{\prime} / E^{0}$, which contains (an isomorphic copy of) $E^{\prime} . p(\bar{B})$ is then $\sigma\left(F^{\prime} / E^{0}, E\right)$-compact. But by the above, $p(\bar{B})=B$, so that $B$ is $\sigma\left(E^{\prime}, E\right)$ compact. This completes the proof.

Remarks. (i) If it is assumed in the theorem that $E$ satisfies condition (**) rather than condition (*), it follows that $E$ is barrelled under $\tau\left(E, E^{\prime}\right)$.

(ii) In either case, (b) may be weakened to:

(b') there is a sequence $\left(z_{n}\right)$ in $E$ such that

$$
z_{n}=\sum_{i \in \Lambda} \beta_{\lambda}^{(n)} x_{\lambda} \quad(n=1,2, \ldots)
$$

and for each $\lambda \in \Lambda$, at least one $\beta_{\lambda}^{(n)}$ is non-zero.

In the proof, let $A_{n}$ be the $\eta$-closed absolutely convex envelope of

$$
\bigcup_{r=1}^{\infty}\left\{\sum_{\lambda \in \phi} \beta_{\lambda}^{(r)} x_{\lambda}: \phi \in \Phi\right\}
$$


and let $M$ be the vector subspace of $F$ generated by $\bigcup_{n=1}^{\infty} A_{n}$. This time $M^{\prime}$ is metrisable under $\tau\left(M^{\prime}, M\right)$ and $q(B)$ is again $\sigma\left(M^{\prime}, M\right)$-compact. The rest of the proof proceeds as before.

Unlike the Fréchet space case (7), the coefficient functionals of a weak extended basis in a locally convex space satisfying condition $\left({ }^{* *}\right)$ may fail to be continuous. However, with the assumption of continuity, the weak basis theorem remains valid.

Theorem 2. Let $E$ be a separated locally convex space with dual space $E^{\prime}$ and suppose that $E$ satisfies condition $\left(^{* *}\right)$. If $\left(x_{\lambda}\right)_{\lambda \in \Lambda}$ is a weak extended $S c h a u d e r$ basis in $E$, then it is an extended Schauder basis for any locally convex topology on $E$ which is finer than $\sigma\left(E, E^{\prime}\right)$ and coarser than $\beta\left(E, E^{\prime}\right)$.

Proof. Let $x_{\lambda}^{\prime}$ be the coefficient functional of $x_{\lambda}(\lambda \in \Lambda)$ and let $\Omega$ denote the set of all finite subsets of the positive integers. It is shown first of all that for each $x \in E$ and each subsequence $\left(x_{\lambda(n)}\right)$ of $\left(x_{\lambda}\right)_{\lambda \in \Lambda}$, the series $\Sigma\left\langle x, x_{\lambda(n)}^{\prime}\right\rangle x_{\lambda(n)}$ is unconditionally Cauchy with respect to $\beta\left(E, E^{\prime}\right)$. If this is not the case there exist an element $z$ of $E$, a $\sigma\left(E^{\prime}, E\right)$-bounded set $B$ and a subsequence $\left(x_{\lambda(n)}\right)$ such that for each positive integer $N$, there exists $\omega \in \Omega$ with $n>N$ for all $n \in \omega$ and $\sum_{n \in \omega}\left\langle z, x_{\lambda(n)}^{\prime}\right\rangle x_{\lambda(n)} \notin B^{0}$. Proceeding inductively, sequences $\left(\omega_{m}\right)$ in $\Omega$ and $\left(x_{m}^{\prime}\right)$ in $B$ can be constructed such that $\omega_{r} \cap \omega_{s}=\varnothing(r \neq s)$ and for all $m$,

$$
\left|\left\langle\sum_{n \in \omega_{m}}\left\langle z, x_{\lambda(n)}^{\prime}\right\rangle x_{\lambda(n)}, x_{m}^{\prime}\right\rangle\right|>1
$$

The set $\left\{x_{m}^{\prime}: m=1,2, \ldots\right\}$ is equicontinuous since $E$ satisfies condition $\left(^{* *}\right)$.

Now consider the set $A=\left\{\sum_{n \in \omega_{m}}\left\langle x_{\lambda(n)}, x_{m}^{\prime}\right\rangle x_{\lambda(n)}^{\prime}: m=1,2, \ldots\right\}$ and let $x \in E$. Since $\Sigma\left\langle x, x_{\lambda}^{\prime}\right\rangle x_{\lambda}$ converges unconditionally under $\sigma\left(E, E^{\prime}\right)$, it follows from (8, Chapter II, Corollary 1 of Theorem $1 ; 9$, Theorem 1$)$ that the set

$$
\left\{\sum_{n \in \omega_{m}}\left\langle x, x_{\lambda(n)}^{\prime}\right\rangle x_{\lambda(n)}: m=1,2, \ldots\right\}
$$

is bounded, and so there exists $M(x) \geqq 0$ such that

or equivalently,

$$
\left|\left\langle\sum_{n \in \omega_{m}}\left\langle x, x_{\lambda(n)}^{\prime}\right\rangle x_{\lambda(n)}, x_{m}^{\prime}\right\rangle\right| \leqq M(x), \quad m=1,2, \ldots,
$$

$$
\left|\left\langle x, \sum_{n \in \omega_{m}}\left\langle x_{\lambda(n)}, x_{m}^{\prime}\right\rangle x_{\lambda(n)}^{\prime}\right\rangle\right| \leqq M(x), \quad m=1,2, \ldots
$$

This last observation shows that $A$ is $\sigma\left(E^{\prime}, E\right)$-bounded, and therefore by condition (**), $A$ is equicontinuous.

The vector subspace of $E$ generated by $\left\{x_{\lambda}: \lambda \in \Lambda\right\}$ is $\sigma\left(E, E^{\prime}\right)$-dense in $E$ and is therefore a dense vector subspace of $E$ for all topologies of the dual pair $\left(E, E^{\prime}\right)$. Thus there exist a finite subset $\phi$ of $\Lambda$ and scalars $\alpha_{\lambda}(\lambda \in \phi)$ such that

$$
z-\sum_{\lambda \in \phi} \alpha_{\lambda} x_{\lambda} \in A^{0}
$$


Also there is a positive integer $N$ such that

Then if $m \geqq N$, by (III),

$$
\phi \cap\left\{\lambda(n): n \in \bigcup_{m=N}^{\infty} \omega_{m}\right\}=\varnothing .
$$

$1 \geqq\left|\left\langle z-\sum_{\lambda \in \phi} \alpha_{\lambda} x_{\lambda}, \sum_{n \in \omega_{m}}\left\langle x_{\lambda(n)}, x_{m}^{\prime}\right\rangle x_{\lambda(n)}^{\prime}\right\rangle\right|$

which contradicts (II).

$$
=\left|\left\langle z, \sum_{n \in \omega_{m}}\left\langle x_{\lambda(n)}, x_{m}^{\prime}\right\rangle x_{\lambda(n)}^{\prime}\right\rangle\right|=\left|\left\langle\sum_{n \in \omega_{m}}\left\langle z, x_{\lambda(n)}^{\prime}\right\rangle x_{\lambda(n)}, x_{m}^{\prime}\right\rangle\right|
$$

It now follows that for each $x \in E$, each subseries of $\Sigma\left\langle x, x_{\lambda}^{\prime}\right\rangle x_{\lambda}$ converges in the $\beta\left(E, E^{\prime}\right)$-completion of $E$, so that by Orlicz's theorem (8, Chapter II, Theorem 9; 9, Theorem), $\Sigma\left\langle x, x_{\lambda}^{\prime}\right\rangle x_{\lambda}$ is unconditionally Cauchy with respect to $\beta\left(E, E^{\prime}\right)$. Since $\Sigma\left\langle x, x_{\lambda}^{\prime}\right\rangle x_{\lambda}$ converges unconditionally to $x$ with respect to $\sigma\left(E, E^{\prime}\right)$, and since $\beta\left(E, E^{\prime}\right)$ has a base of $\sigma\left(E, E^{\prime}\right)$-closed neighbourhoods, $\Sigma\left\langle x, x_{\lambda}^{\prime}\right\rangle x_{\lambda}$ must converge unconditionally to $x$ under $\beta\left(E, E^{\prime}\right)(4$, Section 18 , 4(4)).

The result now follows since the same convergence must take place for any locally convex topology on $E$ which is coarser than $\beta\left(E, E^{\prime}\right)$, and since the coefficient functionals $x_{\lambda}^{\prime}$ are continuous for any locally convex topology on $E$ which is finer than $\sigma\left(E, E^{\prime}\right)$.

\section{REFERENCES}

(1) M. G. Arsove and R. E. Edwards, Generalized bases in topological linear spaces, Studia Math. 19 (1960), 95-113.

(2) M. De WiLde and C. Hovet, On increasing sequences of absolutely convex sets in locally convex spaces, Math. Ann. 192 (1971), 257-261.

(3) T. Husain, Two new classes of locally convex spaces, Math. Ann. 166 (1966), 289-299.

(4) G. KöTHE, Topologische lineare Räume I (Springer-Verlag, Berlin, 1960).

(5) M. Levin and S. SAXoN, A note on the inheritance of properties of locally convex spaces by subspaces of countable codimension, Proc. Amer. Math. Soc. 29 (1971), 97-102.

(6) J. T. MARTI, Extended bases for Banach spaces, Illinois J. Math. 15 (1971), 135-143.

(7) J. T. MARTI, A weak basis theorem for non-separable Fréchet spaces, J. London Math. Soc. 5 (1972), 8-10.

(8) A. P. Robertson, Summation and Integration in Linear Topological Spaces (Ph.D. Dissertation, Cambridge, 1954).

(9) A. P. Robertson, On unconditional convergence in topological vector spaces, Proc. Roy. Soc. Edinburgh Sect. A 68 (1969), 145-157.

10) A. P. Robertson and W. J. Robertson, Topological Vector Spaces (Cambridge University Press, Cambridge, 1963).

UNIVERSITY OF STIRLING 